\title{
Design and Operation of a Photocataytic Reactor: A Study of Dye (Methylene Blue) Removal Process
}

\author{
M. A. Islam, M. Rakib Uddin, M. S. A. Amin, M. I. Haque, M. S. R. Molla \\ Center for Environmental Process Engineering \\ Department of Chemical Engineering and Polymer Science \\ Shahjalal University of Science and Technology, Sylhet-3114
}

\begin{abstract}
The degradation of Methylene Blue (MB) in aqueous solution has been investigated in a photocatalytic reactor under UV radiation and in presence of zinc oxide $(\mathrm{ZnO})$. The photodegradation kinetics follows pseudo-first order reaction. The effect of process parameters such as catalyst loading and initial concentration of $\mathrm{MB}$ on the degradation is investigated. The results show that the degradation rate is affected insignificantly by the increases in the catalyst dosage. Batch test, however, shows that with an increase in the initial concentration the apparent rate constant gradually decreases. The first results with a continuous reactor with a diluted feed solution show inspiring dye removal.
\end{abstract}

\section{INTRODUCTION}

Application of UV radiated semiconductor catalysts for the removal of organic compounds from water has been a topic of interest for last few decades ${ }^{1-3}$. Over the years, a large number of semiconductors have been used as photocatalysts. The most commonly studied photocatalysts are $\mathrm{TiO}_{2}, \mathrm{ZnO}$ and $\mathrm{CdS}{ }^{4-6}$. Among the various semiconductors the $\mathrm{TiO}_{2}$ is generally considered to be the best photocatalyst and has been widely used for the treatment of water from a number of organic pollutants ${ }^{7-9}$. However, widespread use of $\mathrm{TiO}_{2}$ is uneconomical for large-scale water treatment operations. It has become an imperative to find an alternative to $\mathrm{TiO}_{2}$.

The photocatalytic oxidation technology has appeared to be a highly effective technology for the complete degradation of organic micro-pollutants (dyes) in water, utilizing sunlight and UV radiation as energy sources ${ }^{4}$,

${ }^{10}$. Photocatalytic reactor design, however, remains one of the most challenging options of research in recent times ${ }^{11}$. Mozia ${ }^{12}$ reviewed some membrane reactors with description and characterization. In the present investigation, a reactor is prepared and photocatalytic degradation of $\mathrm{MB}$ in the presence of $\mathrm{ZnO}$ and with $\mathrm{UV}$ light as the illuminant has been reported. The effect of the parameters such as catalyst dosage and the initial concentration on the degradation of $\mathrm{MB}$ has been examined and the results obtained are discussed. Finally, a schematic diagram has been proposed for continuous degradation of organic pollutants with a photocatalytic reactor unit designed and tested in the present work.

\section{MATERIALS AND METHOD}

\subsection{Materials}

Commercial $\mathrm{ZnO}$ supplied by LUBA CHEMIE (which is not a standard semiconductor photocatalyst, rather used as filler and pigment) was used as catalyst powder. MB (MERK) was used as representative dye. All solutions were prepared using Double Distilled water. UV lamp $(230 \mathrm{~V}, 50 \mathrm{~Hz}, \mathrm{BDH})$ was used as illuminant.

\subsection{Photocatalytic Batch Reactor Setup}

Photocatalytic degradation of $\mathrm{MB}$ in presence of UV light as a radiation source was carried out in a reactor designed for the purpose. Air was blown into the reactor at a constant rate to accelerate the oxidation process. Also, the reactor was equipped with magnetic stirrer. The UV lamp was placed on the top of the reactor and the whole system was kept in a wooden casing with light cork sheet insulation so that the UV ray cannot get out.

\subsection{Experiment procedure}

The experiments were conducted with a $200 \mathrm{~mL}$ of dye solution for each reaction with varying catalyst dosages and initial dye concentrations. The solution was continuously aerated. Mechanical stirring was conducted for dispersing the precipitating catalyst particles back to the bulk of the dye solution. Samples were taken every ten minutes and centrifuged at 800 rpm for five minutes to separate catalyst powders out from the sample solution. The absorbance of the dye solution was measured with a UV-Spectrophotometer (UV-1650, SHIMADZU) at $664.5 \mathrm{~nm}$.

\footnotetext{
* Corresponding Author: M. S. A. Amin 


\section{RESULTS AND DISCUSSION}

\subsection{Preliminary Photodegradability of MB}

The preliminary test was conducted at different operational conditions: a) Simple adsorption of the dye molecules on the catalyst surface, b) photodegradation of the dye solution in absence of catalyst particles and c) photodegradation under UV radiation with varying catalyst dosages. The initial concentration was kept constant at 10ppm in all the experiments. The results of the test are presented in Figure. For an experimental period of 50 minutes, the dye removal through adsorption on the catalyst surface was below the detection limit of the method of dye-measurement. Also, the dye shows very poor degradability under UV light without $\mathrm{ZnO}$. But in presence of $\mathrm{ZnO}$ particles under UV radiation, the degradation rate is very high and $50 \%$ of the dye is degraded within the first ten minutes of experimentation. The degradation curves with the photocatalyst concentration of $0.25-1.0 \mathrm{~g} / \mathrm{L}$ are undistinguishable for practical purposes showing little difference in the effect of catalyst concentrationvariation within the dosage-range under investigation.

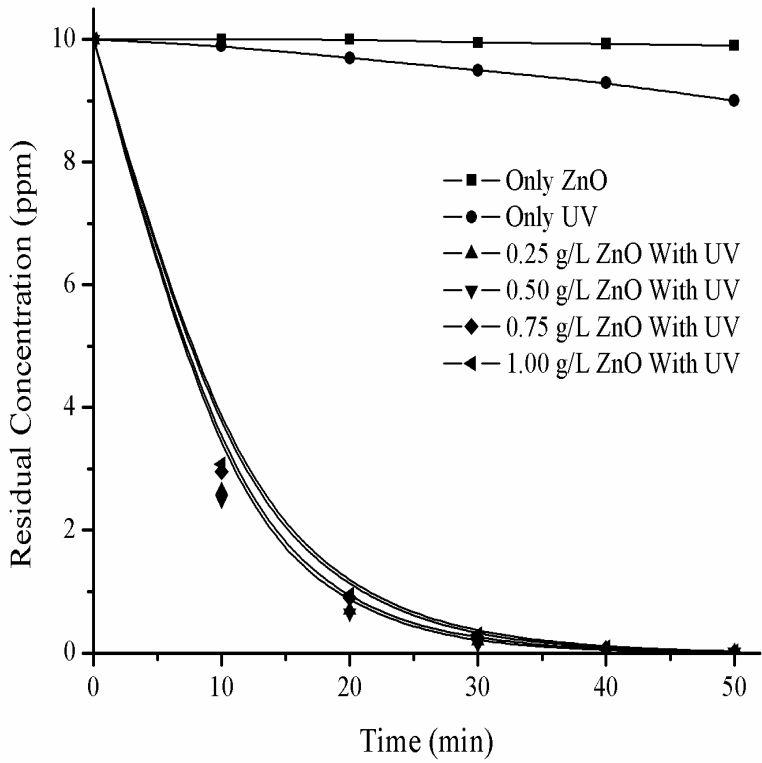

Fig. 1: Dye (MB) removal at various conditions (with catalyst but without radiation, with radiation only, and with radiation in presence of catalyst)

The photodegradability data with varying catalyst concentration was fitted to the pseudo-first order chemical kinetics model (Eq. 1).

$$
C=C_{0} \exp (-k t)
$$

Where $C$ and $C_{0}$ respectively are the residual and initial concentration (ppm) of the dye, $k\left(\mathrm{~min}^{-1}\right)$ is the apparent rate constant of photodegradation and $t$ (min) is the operational time

As seen in the Figure, the Eq.(1) satisfactorily describes the photodegradation kinetics of the dye in the catalyst concentration-range of 0.25 to $1.0 \mathrm{~g} / \mathrm{L}$, and the fitted value of the apparent rate constant $k$ was found to lie in the narrow range of $(0.12-0.14) \mathrm{min}^{-1}$; with the highest value for the catalyst concentration of $0.5 \mathrm{~g} / \mathrm{L}$. Hence, $0.5 \mathrm{~g} / \mathrm{L}$ is taken to be the optimum catalyst concentration for the photocatalytic degradation of MB under the condition of experimentation. Such observation, however, contradicts with the expectation that the higher catalyst concentration would give higher $k$-value. This unexpected result can be attributed to the counterbalance of the effect of availability of active sites on the catalyst surface and the penetration of UV light into the suspension. The total active surface area increases with increasing catalyst dosage. But at the catalyst dosage above $0.5 \mathrm{~g} / \mathrm{L}$, there is a decrease in UV light penetration due to screening effect of excess catalyst particle in the solution ${ }^{13}$ and as a result, the increase in the catalyst concentration shows insignificant effect on the degradation rate.

\subsection{Effect of initial concentration}

The photocatalytic degradation of MB was carried out with initial concentrations $2-10 \mathrm{ppm}$ and the catalyst dosage constant at $0.5 \mathrm{gm} / \mathrm{L}$. The degradation data are fitted to rearranged form of the pseudo-first order reaction kinetic model (Eq.2) and are presented in Figure 2.

$-\ln \left(C / C_{0}\right)=k t$

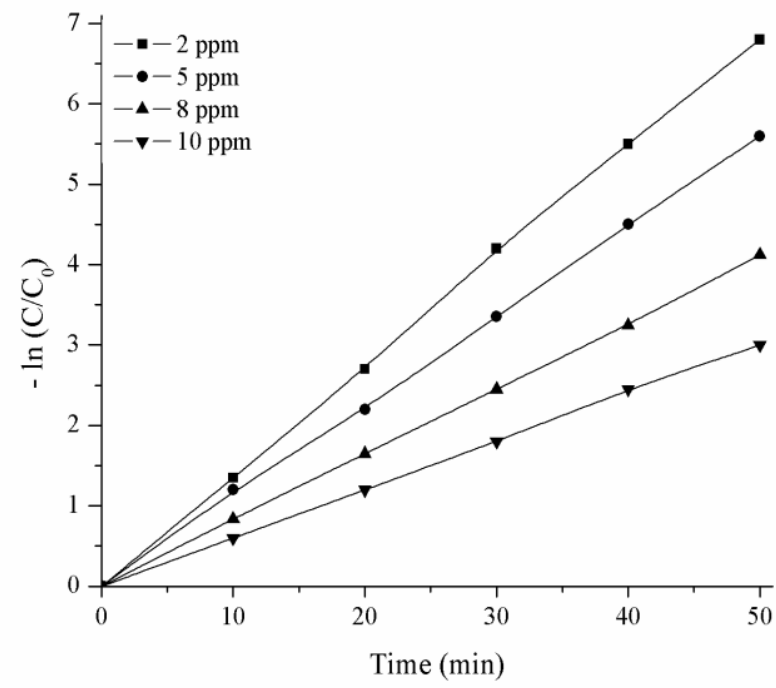

Fig.2: $-\ln \left(C / C_{0}\right)$ vs $t$ plot for different initial dye concentration $(2-10 \mathrm{ppm})$ with the catalyst concentration of $0.5 \mathrm{~g} / \mathrm{L}$ 
It is evident from the Figure that the photodegradation kinetics of $\mathrm{MB}$ is satisfactorily described by the pseudo-first order reaction kinetic model. The apparent rate constant $k$ (determined from the slope of the straight lines in Figure 2), however, is very sensitive to initial concentration. The fitted value of $k$ is presented in Figure 3 as a function of initial concentration. Unlike the catalyst concentrations, the variation of which does not cause significant change in reaction rate, the effect of initial concentration on the reaction rate is quite distinct.

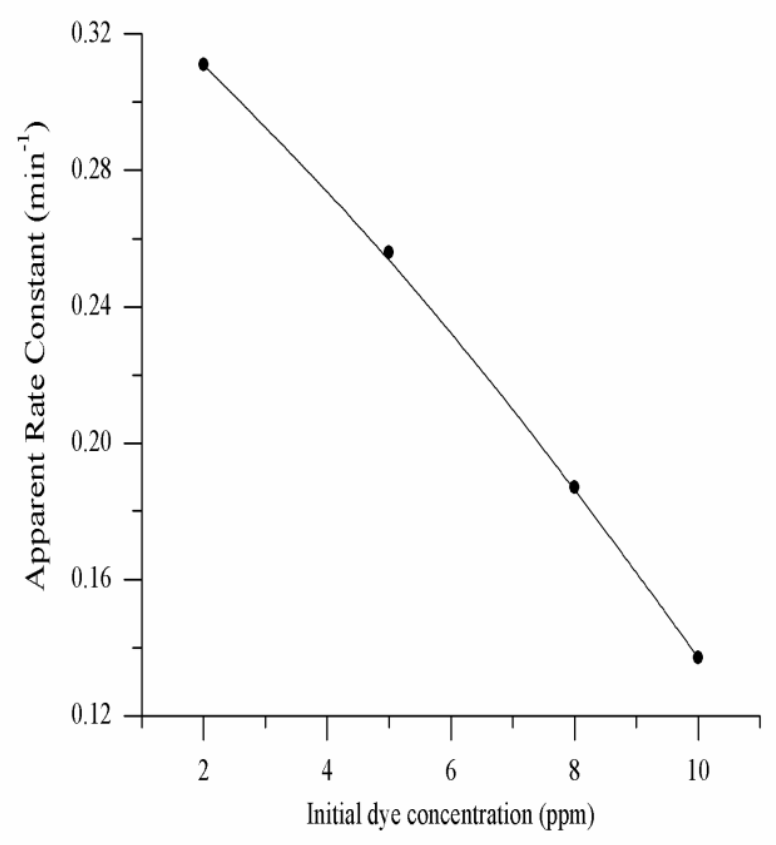

Fig. 3: Effect of initial dye concentration $C_{0}$ on the apparent rate constant $k$

The reaction rate constant decreases with increasing dye concentration. Such behavior may be explained with the presumption that as initial concentration increases, the more and more organic substances are adsorbed on the surface of $\mathrm{ZnO}$. This reduces the generation of hydroxyl radicals, since there are only a fewer active sites for the adsorption of hydroxyl ions and the generation of hydroxyl radicals for catalytic reaction. Further, as the concentration of MB solution increases, the photons get intercepted before they can reach the catalyst surface. Hence, the absorption of photons by the catalyst decreases, and consequently the degradation rate is reduced ${ }^{14}$.

\subsection{Reusability of Photocatalyst}

Reusability of $\mathrm{ZnO}$ photocatalyst for the degradation of MB was evaluated with the following procedure: The solution resulting from the photocatalytic degradation of $\mathrm{MB}$ was filtered, washed and the photocatalyst particles were dried. The dried catalyst samples were used for the degradation of $\mathrm{MB}$ under similar conditions. It was found that the photocatalyst $\mathrm{ZnO}$ could be used repeatedly without any treatment and the catalytic activity of reused catalyst was found to be in the same range as that of the virgin catalyst even after five successive batch reactions.

\subsection{Photocatalytic Reactor Setup for continuous operation}

For the continuous degradation of $\mathrm{MB}$ a rectangular box shaped reactor with dimension $40 \times 10 \times 10 \mathrm{~cm}^{3}$ was used. The reactor was made of mirror glass. Air was bubbled through the dye solution at a constant rate of $150 \mathrm{~mL} / \mathrm{min}$ to accelerate the oxidation process. Simultaneously, the whole liquid mass was stirred mechanically with three magnetic stirrers placed underneath the reactor. The UV lamp was placed on top of the reactor and the whole system was kept in a wooden casing with light cork sheet insulation so that the UV ray cannot get out. Catalyst coated aluminum plates were used, where synthetic adhesive resin was used as a binder for the coating. Feed was charged into the reactor by using a pump from a feed tank. Liquid level sensor was used to control the flow rate. As observed in batch study, the photo degradation rate decreases with the increase in the dye concentration. For that reason, $80 \%$ of the treated water was recycled to mix with a feed solution of $10 \mathrm{ppm}$. The first results of the experimentations with the continuous reactor with the chosen residence time equal to $1 \mathrm{~h}$ were quite satisfactory. The experimental set up for the investigation of continuous degradation process is shown in Fig. 4.

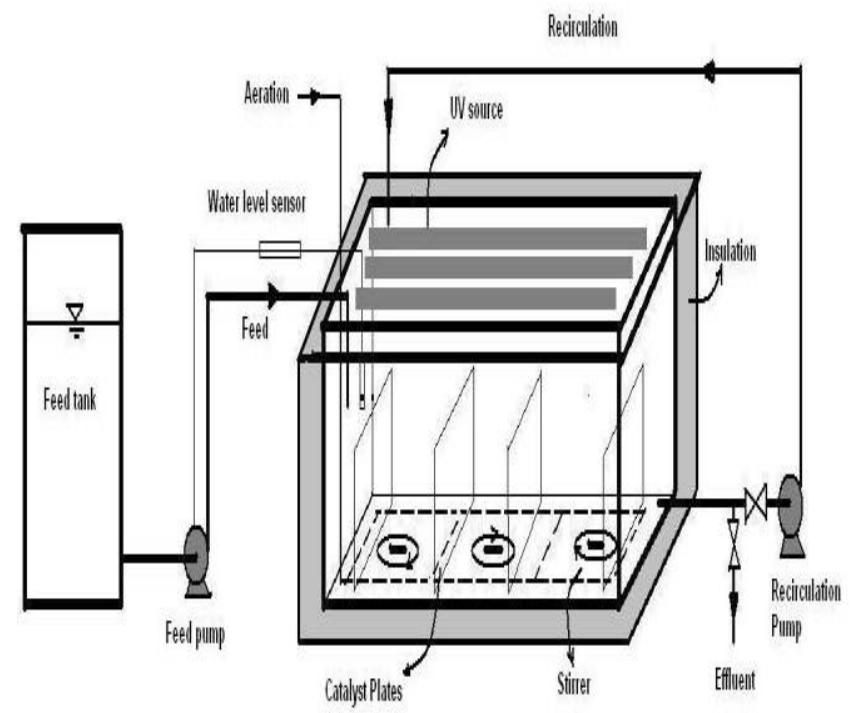

Fig. 4: Photocatalytic Reactor for continuous process 


\section{CONCLUSIONS}

Commercial pigment/filler grade $\mathrm{ZnO}$ is an effective photocatalyst for the degradation of MB under UVradiation. The photodegradation kinetics follows pseudo-first order chemical kinetics. The effect of catalyst dosage in the range of $0.25-1.0 \mathrm{~g} / \mathrm{L}$ is insignificant on the reaction rate. With the increase in the initial concentration in the batch tests, the apparent rate constant is found to decrease monotonously. A continuous reactor was designed and tested with the informations collected from the batch tests. The first results from the continuous reactor are quite inspiring.

Acknowledgement: The financial support from the SUST Research Grant (2012-13) for conducting the research is highly appreciated.

\section{REFERENCES}

[1] Akyol, A., Yatmaz, H.C. and Bayramoglu M. (2004), Photocatalytic decolorization of Remazol Red RR in aqueous $\mathrm{ZnO}$ suspensions, Appl. Catal. B: Environ, 54: 1924

[2] Ahmed, S., Rasul, M.G., Martens, W. N., Brown R., Hashib, M.A. (2010), Heterogeneous photocatalytic degradation of phenols in wastewater: A review on current status and developments, Desalination, 261: 3-18

[3] Rao, A. N., Sivasankar, B., Sadasivam, V. (2009), Kinetic study on the photocatalytic degradation of salicylic acidusing $\mathrm{ZnO}$ catalyst, Journal of Hazardous Materials, 166: 1357-1361

[4] Uddin, M.J., Cesano, F., Bonino, F., Bordiga, S., Spoto, G., Scarano, D. and Zecchina, A., (2007) Photoactive $\mathrm{TiO}_{2}$ films on cellulose fibres: synthesis and characterization, $\mathrm{J}$. Photochem. Photobiol. A-Chem., 189: 286 294

[5] Akyol, A., Bayramoglu, M. (2005), Photocatalytic degradation of Remazol Red
F3B using $\mathrm{ZnO}$ catalyst, Journal of Hazardous Materials, 124(B): 241-246

[6] Akpan, U.G., Hameed, B.H. (2009), Parameters affecting the photocatalytic degradation of dyes using $\mathrm{TiO}_{2^{-}}$ basedphotocatalysts: A review, Journal of Hazardous Materials, 170: 520-529

[7] Ahmed, S., Rasul, M.G., Brown, R., Hashib, M.A. (2011), Influence of parameters on the heterogeneous photocatalytic degradation of pesticides and phenolic contaminants in wastewater: A short review, Journal of Environmental Management, 92: 311-330

[8] Han, F., Kambala, V. S.R., Srinivasan, M., Rajarathnam, D., Naidu, R. (2009), Tailored titanium dioxide photocatalysts for the degradation of organic dyes in waste water treatment: A review, Applied Catalysis A: General, 359: 25-40

[9] Konyar, M., Cengiz, Y.H., Öztürk, K., (2012), Sintering temperature effect on photocatalytic efficiencies of $\mathrm{ZnO} / \mathrm{TiO}_{2}$ composite plates, Applied Surface Science, 258: 7440-7447

[10] Fox, M.A. and Dulay, M.T. (1993), Heterogeneous photocatalysis, Chem. Rev., 93: $341-357$

[11] Chong, M.N., Jin, .B, Chow, C.W., Saint, C.(2010), Recent Developments in photocatalytic water treatment technology: A review, Water Res., 44(10):2997-3027

[12] Mozia, S. (2010), Photocatalytic membrane reactors (PMRs) in water and wastewater treatment: A review, Separation and Purification Technology, 73: 71-91

[13] Sobana N. and Swaminathan M. (2007), The effect of operational parameters on the photocatalytic degradation of acid red 18 by ZnO, Sep. Purif. Technol., 56: 101-107

[14] Behnajady M.A ., Modirshahla N. and Hamzavi R. (2006), Kinetic study on photocatalytic degradation of C.I. Acid Yello 23 by $\mathrm{ZnO}$ photocatalyst, J. Hazard. Mater. 133: $226-232$ 